Rev. salud pública. 12 (2): 239-249, 2010

\title{
Tabaquismo y supervivencia estudiantil en la Universidad Santiago de Cali. 2004-2007
}

\section{Smoking and student survival at Universidad Santiago de Cali, 2004-2007}

Luis A. Tafur-Calderón ${ }^{1}$, Juan C. Millán-Estupiñan ${ }^{1}$, Helmer Zapata-Ossa $^{1}$, Gustavo A. Ordoñez-Arana² y Jesús M. Varela ${ }^{1}$

1 Facultad de Salud, Universidad Santiago de Cali, Colombia. lutafur@yahoo.com, juancarlosmillane@yahoo.com, hdejzapata@emcali.net.co, jmvarela@usc.edu.co

2 Asociación Latinoamericana de Neumología. ordonezgustavoa@yahoo.com

Recibido 7 Julio 2009/Enviado para Modificación 31 Enero 2010/Aceptado 12 Abril 2010

\section{RESUMEN}

Objetivos Este artículo presenta los resultados del seguimiento de los estudiantes ingresados y matriculados en la Universidad Santiago de Cali USC en el segundo semestre de 2004. El propósito fue determinar la influencia del tabaquismo, el programa académico y el costo de la matrícula en la supervivencia como estudiantes en la universidad durante un período de tres años 2004-2007.

Método El estudio fue de cohorte prospectiva sobre el grupo de 970 estudiantes que ingresaron en el año 2004. Para determinar la relación entre las variables independientes y la supervivencia universitaria, se realizó análisis de supervivencia a través de la regresión de Cox.

Resultados Los resultados del modelo establecieron asociaciones entre tabaquismo y Facultad descartando costos de matrícula. El riesgo de deserción es mayor entre estudiantes de la facultad de Salud ajustado por tabaquismo $(R R=1,3(1,1-$ 1.5)). De manera similar el riesgo es mayor en los fumadores ajustado por la Facul$\operatorname{tad}(\mathrm{RR}=1,2(1,1-1,4))$.

Conclusiones Se encontró que los fumadores habituales permanecen menos en la universidad que los no fumadores. La supervivencia universitaria fue mayor en los programas académicos diferentes al área de la salud.

Palabras Clave: Tabaquismo, análisis de supervivencia, deserción escolar, tabaco. (fuente: DeCS, BIREME).

\section{ABSTRACT}

Objective This article presents the results of monitoring students who enrolled at Universidad Santiago de Cali (USC) during the second half of 2004. Its purpose was to determine the influence of smoking, the academic programme and the cost of enrolment on student survival over a three-year period (2004-2007).

Method The study involved a prospective cohort of 970 students who entered the university in 2004. Cox regression was used for survival analysis to determine the relationship between independent variables and university stay.

Results The results of this model established associations between smoking and 
department with survival in the university, but discarded association with the cost of enrolment. The risk of university desertion was higher amongst students from the Health faculty adjusted for smoking (RR $=1.277(1.121-1.455))$. Similarly, the risk of desertion was higher in smokers adjusted by faculty $(R R=1.194$ (1.026-1.390).

Conclusions It was found that habitual smokers had shorter university stay than nonsmokers. University stay was longer in students enrolled in academic programmes other than health.

Key Words: Smoking, university survival (source: MeSH, NLM).

$\mathrm{D}$ esde 2003 el Grupo de Investigaciones en Salud Respiratoria de la Facultad de Salud de la Universidad Santiago de Cali (USC) ha realizado estudios del hábito de fumar entre los estudiantes recién ingresados a la institución (1,2). La hipótesis inicial fue que el ambiente universitario incrementa el consumo del cigarrillo e incita a la iniciación del mismo. Los estudios concluyeron que no hay relación entre el ambiente universitario y el tabaquismo. En el estudio de seguimiento que se inició en 2004 se ha observado una disminución del número de estudiantes al avanzar en los semestres académicos, con la hipótesis que los fumadores permanecen menos tiempo en la universidad que los no fumadores, este estudio plantea determinar la asociación entre tabaquismo y la supervivencia estudiantil en los programas académicos.

La supervivencia en los programas académicos se encuentra asociada a factores socioeconómicos, afinidad del estudiante con la carrera en la que se matriculó, logro académico, oportunidades económicas del estudiante (tanto para el pago de la matrícula como para su sostenimiento personal), capital cultural previo y ambiente familiar de estudio, los cuales inciden en el rendimiento académico (3). Korhonen y col., encontraron asociación entre bajo rendimiento académico y consumo de alcohol, fumar cigarrillos y abuso de otras sustancias (4).

El tabaquismo se considera un problema de salud pública nacional, que genera altos costos a los sistemas de salud. El costo de la atención médica de las Enfermedades asociadas al Tabaquismo se da en función de la supervivencia. Sólo considerando los costos médicos directos, el gasto anual en la atención médica de las enfermedades atribuibles al tabaco representó alrededor del 0,7 \% del total del PIB de Colombia en 2004 (5). Un estudio realizado en el Estado de Morelos, México, en una cohorte de estudiantes del ámbito escolar de los niveles de secundaria, bachillerato y licenciatura, entre los años 1998 a 2001 encontró relación entre el bajo desempeño escolar y la persistencia del tabaquismo (6). El bajo rendimiento escolar está relacionado con un alto riesgo 
de ser fumador habitual, independiente de otras covariables (7). Hu y col.(1998) determinaron que existe relación entre los bajos logros académicos durante la adolescencia y la presencia del hábito (8).

El Grupo de Investigaciones en Salud Respiratoria "GISAR" de la Universidad Santiago de Cali tiene como uno de sus objetivos estudiar el comportamiento de los factores de riesgo de enfermedades respiratorias en la población, con énfasis en los estudiantes universitarios. Desde 2003 viene realizando estudios transversales de medición de la prevalencia del tabaquismo en los estudiantes recién ingresados en los años 2003(1) y 2004 (2) y de seguimiento al grupo que ingresó en 2004 mediante medición de la prevalencia en los grupos matriculados en tercero, quinto y séptimo semestre de los diversos programas académicos que ofrece la Universidad.

Este artículo presenta los resultados del seguimiento de los estudiantes ingresados y matriculados en la USC en el segundo semestre de 2004, y la influencia del tabaquismo en la supervivencia en la universidad en los semestres tercero (2005), quinto (2006) y séptimo (2007), excluyendo del análisis a los estudiantes matriculados en los programas académicos de duración inferior a siete semestres (Tecnologías en Mecánica dental y Atención Prehospitalaria).

\section{MATERIALES Y METODOS}

Este estudio corresponde a una cohorte prospectiva de la totalidad de estudiantes de la USC que ingresaron a primer semestre en el año 2004 a los cuales se les hizo seguimiento durante el período 2004 a 2007. La población de estudio debió cumplir los siguientes criterios de inclusión: a. Estudiantes que ingresaron a primer semestre que estuvieran matriculados académica y financieramente en los diferentes programas de pregrado que ofrece la Universidad; b. Estudiantes de la jornada diurna. La encuesta fue elaborada con base en los criterios de la Organización Mundial de la Salud (OMS) (9) y tiene preguntas relacionadas con las características sociodemográficas de las personas como género, estado civil, Facultad y programa académico. La clasificación frente al cigarrillo como fumadores, habituales o esporádicos, y no fumadores. Siguiendo los criterios de la OMS se definió: fumador habitual quien fuma 1 o más cigarrillos por día o 5 o más por semana en el último año. Fumador esporádico es quien fuma 4 o menos cigarrillos por semana. Teniendo como base los resultados de los estudios previos en los cuales se observaron diferencias entre la prevalencia del tabaquismo en los estudiantes de los programas de salud y el resto, los estudiantes fueron clasificados en 2 grupos, 
aquellos que pertenecían a la facultad de salud "programas de salud" y los que pertenecían a programas distintos a esa facultad "no salud". Los costos de matricula se obtuvieron de información suministrada por el Departamento Financiero de la Universidad y se agruparon de la siguiente manera: valores menores de dos millones de pesos colombianos (US \$ 1000 ), valores entre dos y tres millones (US \$2000-3000) y más de tres millones (US \$ +3000).

Para la recolección de la información los estudiantes se visitaron por los investigadores en los salones de clase, quienes hicieron énfasis en el manejo confidencial de la información así como en la necesidad de la veracidad en las respuestas. Se informó a los estudiantes que el estudio no tendría relación con las notas académicas ni con su permanencia en la Universidad. Seguidamente se les suministro el instrumento y el consentimiento informado. Se solicitó a la Secretaría Académica de la Universidad el listado de los estudiantes registrados en ella como matriculados formalmente en los semestres $1,3,5$ y $7^{\circ}$ de los años 2004, 2005, 2006 y 2007 respectivamente. Posteriormente, se revisó el listado de los estudiantes matriculados en primer semestre de 2004 comparando con la presencia de ellos en los semestres siguientes. Una vez establecidos los grupos entrevistados y matriculados, se comparó con los datos de tabaquismo obtenidos en las encuestas realizadas en 2005, 2006 y 2007.

La base de datos se construyó en el programa Excel 8.0 y los análisis estadísticos se realizaron en el programa SPSS versión 16.0, serie 9811696. Se realizó un análisis univariado a través de tablas de frecuencia comparando los dos grupos de interés.

Como variable de exposición se tomó el tabaquismo con las categorías habitual, esporádico o no fumador, y el resultado, el permanecer como estudiante matriculado en los programas en los diferentes semestres académicos en los cuales se hizo seguimiento. El análisis del tiempo de permanencia en la Universidad (supervivencia universitaria) se realizó mediante la técnica de tabla de mortalidad (10). Se consideró como evento "falla: la deserción universitaria" y evento "no falla: supervivencia universitaria"; en los semestres de los años 2004-2007 como períodos o intervalos de observación. En cada intervalo de observación, se identificaron los estudiantes matriculados con el propósito de calcular la probabilidad de que un evento terminal, es decir, una deserción hubiera tenido lugar dentro de ese lapso. Las probabilidades estimadas para cada intervalo se utilizaron para estimar la probabilidad global que el evento tenga lugar en diferentes puntos temporales del período. Para el análisis se asumió que los retiros de los estudiantes se distribuyen homogéneamente en 
los intervalos de estudio, es decir, el número de estudiantes a riesgo en un intervalo es igual al número de estudiantes que entran menos la mitad del número que se pierde o retira del intervalo. Los resultados se complementaron con gráficos para la variables supervivencia $S(t)$ y de la variable riesgo $h(t)$,

Para establecer las posibles asociaciones estadísticas entre la supervivencia universitaria con el consumo de tabaco y demás variables de interés, se realizaron análisis comparativos de curvas de supervivencia a través de la prueba estadística test de Mantel-Haenszel también llamado test Log-rank (10).

Se realizó una regresión de Cox, teniendo en cuenta posibles factores asociadas a la deserción como facultad a la cual pertenece, costos de la matricula, género y estado civil. Para efectos de este análisis la variable tabaquismo se categorizó en fumador y no fumador.

El estudio se rigió por lo establecido en la resolución 008430 de 1993 del Ministerio de Salud de Colombia. Fue presentado ante el Comité de Ética de la USC, el cual dio un concepto favorable.

\section{RESULTADOS}

En el segundo semestre de 2004 se encuestaron 1186 estudiantes de los cuales se incluyeron en este estudio $970(81,8 \%)$ que se encontraban matriculados académica y financieramente.

Según los resultados de la Tabla 1, la supervivencia de la cohorte bajo estudio a los tres años de seguimiento fue del $29 \%$. Este comportamiento fue similar tanto en hombres como en mujeres (Estadístico de Wilcoxon $(G e h a n)=1,329 ; p=0,249)$. En relación con el estado civil, se observa que la supervivencia para los dos primeros años es ligeramente mayor en los estudiantes casados, sin embargo al final del período no se identificaron diferencias significativas en las diferentes categorías. (Estadístico de Wilcoxon $($ Gehan $)=4,1 ; \mathrm{p}=0,249)$.

Se identificaron diferencias estadísticamente significativas del tiempo de supervivencia universitaria en relación con la facultad de estudio. La supervivencia fue estadísticamente mayor en los programas pertenecientes a las facultades diferentes a la de salud (Estadístico de Wilcoxon (Gehan) $=17,3$; $\mathrm{p}=0,000$ ). Al evaluar costo de matrícula y supervivencia universitaria, se identificó 
que a menor costo de matrícula mayor es la supervivencia universitaria (Estadístico de Wilcoxon (Gehan) $=12,5 ; \mathrm{p}=0,002$ ), siendo las diferencias estadísticamente significativas entre el menor valor de matrícula y los otros grupos $(\mathrm{p}<0,05)$.

Tabla 1. Permanencia de los estudiantes que ingresaron a la USC en el año 2004, según características sociodemográficas. 2004-2007

\begin{tabular}{|c|c|c|c|c|c|c|c|c|}
\hline \multirow{2}{*}{$\begin{array}{l}\text { Características } \\
\text { Sociodemográficas }\end{array}$} & \multicolumn{2}{|c|}{2004} & \multicolumn{2}{|c|}{2005} & \multicolumn{2}{|c|}{2006} & \multicolumn{2}{|c|}{2007} \\
\hline & No. & $\%$ & No. & $\%$ & No. & $\%$ & No. & $\%$ \\
\hline \multicolumn{9}{|l|}{ Sexo } \\
\hline Hombres & 325 & 100 & 206 & 63 & 140 & 43 & 92 & 28 \\
\hline Mujeres & 645 & 100 & 440 & 68 & 297 & 46 & 191 & 30 \\
\hline \multicolumn{9}{|l|}{ Estado Civil } \\
\hline Soltero & 912 & 100 & 608 & 67 & 408 & 45 & 267 & 29 \\
\hline Casado & 35 & 100 & 27 & 77 & 21 & 60 & 10 & 29 \\
\hline Unión libre & 13 & 100 & 7 & 54 & 6 & 46 & 4 & 31 \\
\hline \multicolumn{9}{|l|}{ Facultades } \\
\hline No Salud & 384 & 100 & 267 & 69,5 & 188 & 49 & 150 & 38,1 \\
\hline Salud & 586 & 100 & 379 & 64,7 & 249 & 42,5 & 133 & 22,7 \\
\hline \multicolumn{9}{|c|}{ Costos Matrícula (millones de pesos colombianos) } \\
\hline$<2$ & 50 & 100 & 45 & 90 & 27 & 54 & 22 & 44 \\
\hline $2-3$ & 532 & 100 & 360 & 68 & 243 & 46 & 165 & 31 \\
\hline$>3$ & 392 & 100 & 245 & 63 & 167 & 43 & 96 & 24 \\
\hline Total & 970 & 100 & 620 & 67 & 437 & 45 & 283 & 29 \\
\hline
\end{tabular}

En la Tabla 2 se muestra el número de ingresados en 2004 que permanecieron matriculados en los programas profesionales según la Secretaría Académica de la Universidad distribuidos por hábito de fumar.

Tabla 2. Estudiantes ingresados en el año 2004 y que permanecieron en la Universidad durante los años 2005, 2006 y 2007 según el habito

\begin{tabular}{lrrrrrrrr}
\hline \multirow{2}{*}{ Clasificación de fumador } & \multicolumn{2}{c}{2004} & \multicolumn{2}{c}{2005} & \multicolumn{2}{c}{2006} & \multicolumn{2}{c}{2007} \\
\cline { 2 - 9 } & No. & $\%$ & No. & $\%$ & \multicolumn{1}{c}{ No. } & $\%$ & \multicolumn{1}{c}{ No. } & \multicolumn{1}{c}{$\%$} \\
\hline no fumadores & 754 & 100 & 511 & 67,8 & 362 & 48,0 & 233 & 30,9 \\
Fumadores & 216 & 100 & 135 & 62,5 & 75 & 34,7 & 50 & 3,2 \\
Esporádicos & 118 & 100 & 79 & 67,0 & 46 & 39,0 & 31 & 26,3 \\
Habituales & 98 & 100 & 56 & 57,1 & 29 & 29,6 & 19 & 19,4 \\
\hline \multirow{2}{*}{ Total } & 970 & 100 & 646 & 66,6 & 437 & 45,1 & 283 & 29,2 \\
\hline
\end{tabular}

La supervivencia de permanencia universitaria a los tres años de seguimiento en los estudiantes que manifestaron no haber fumado en su vida al momento de la encuesta, fue del $31 \%$, significativamente mayor que de aquellos clasificados como fumadores habituales o esporádicos, la cual fue de $19 \%$ (Estadístico de Wilcoxon (Gehan) $=8,96 ; \mathrm{p}=0,0027$ ). No se encontró diferencia estadísticamente significativa entre no fumadores y fumadores esporádicos. 
En las Figuras 1 y 2 se presentan los resultados del análisis de supervivencia universitaria según hábito de fumador y no fumador, separados por tipo de facultad. El análisis de supervivencia universitaria según hábito, no mostró diferencias estadísticamente significativas en los programas de la facultad de salud, (test de Wilcoxon (Gehan) Statistic de 3,166; p=0,205), mientras que en los "programas de no salud" la supervivencia universitaria fue mayor en los no fumadores comparado con los fumadores, las diferencias fueron estadísticas significativas (test de Wilcoxon (Gehan) Statistic de 12,974; $\mathrm{p}=0,002$ ).

Figura 1. Supervivencia universitaria en la cohorte de estudiantes 2004-2007 de la Facultad de Salud de la Universidad Santiago de Cali, según hábito de consumo de tabaco

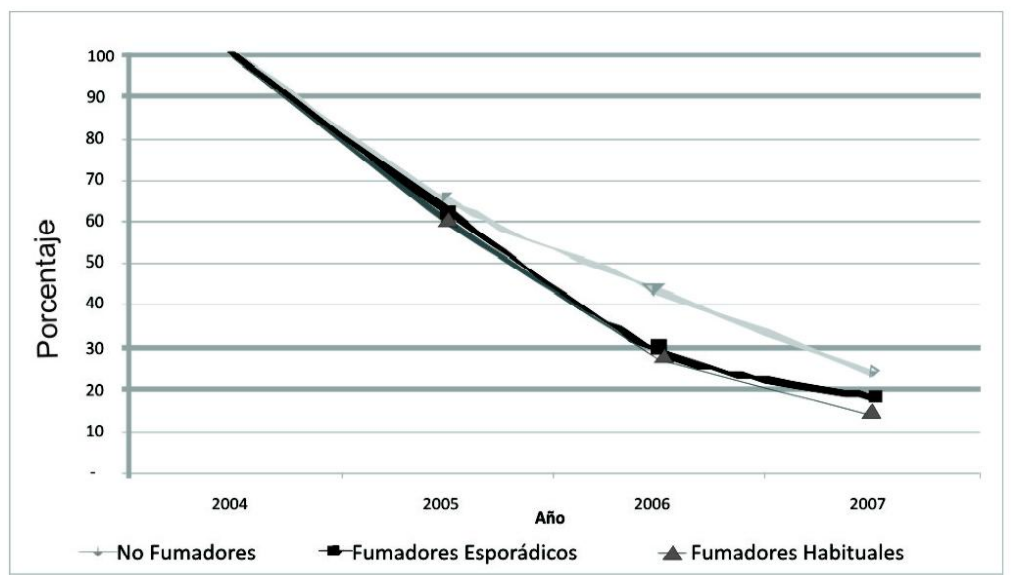

Figura 2. Supervivencia universitaria en la cohorte de estudiantes 2004-2007 de las Facultades diferentes a Salud de la Universidad Santiago de Cali, según hábito de tabaco

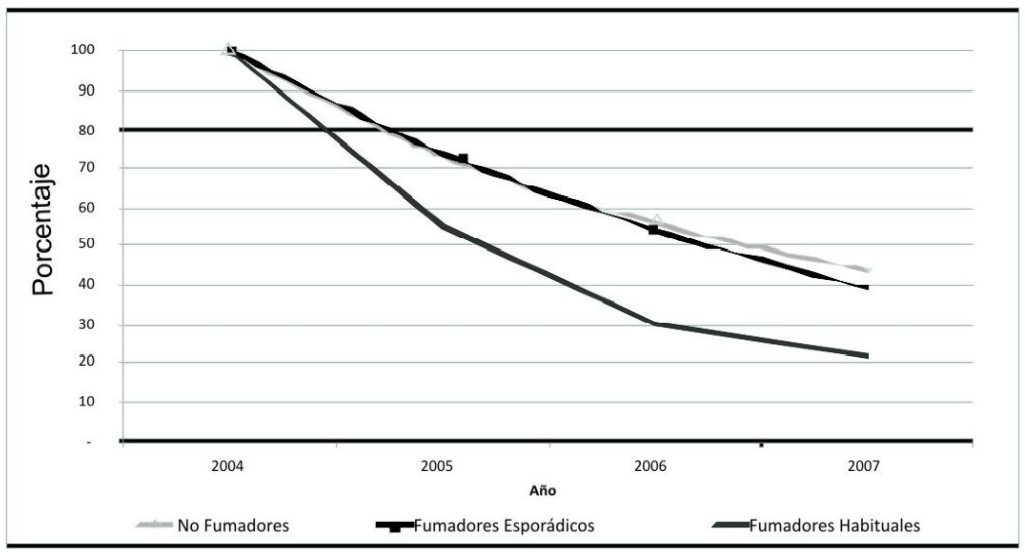


En el análisis bivariado se identificaron algunos factores asociados entre los cuales están tabaquismo, facultad y costo de matrícula. Seguidamente se procedió a la aplicación de un modelo de regresión el cual arrojó los resultados de la Tabla 3.

Los resultados del modelo establecieron asociaciones entre tabaquismo y facultad descartando costos de matrícula. El riesgo de deserción es mayor entre estudiantes de la facultad de Salud ajustado por tabaquismo $(\mathrm{RR}=1,3$ $(1,1-1,5))$. De manera similar el riesgo es mayor en los fumadores ajustado por la Facultad $(\mathrm{RR}=1,2(1,1-1,4))$.

Tabla 3. Resultados de la regresión de Cox para la variable permanencia universitaria según tabaquismo, facultad y costo de matrícula

\begin{tabular}{|c|c|c|c|c|c|c|c|c|c|}
\hline \multirow{2}{*}{\multicolumn{2}{|c|}{ Variables }} & \multirow[t]{2}{*}{ B } & \multirow[t]{2}{*}{ EE } & \multirow[t]{2}{*}{ EW } & \multirow[t]{2}{*}{ GL } & \multirow[t]{2}{*}{$\mathrm{S}$} & \multirow[t]{2}{*}{$e^{\beta}$} & \multicolumn{2}{|c|}{$\begin{array}{l}\text { Intervalo de } \\
\text { Confianza al } \\
95 \% \text { para } e^{\beta}\end{array}$} \\
\hline & & & & & & & & Inferior & Superior \\
\hline \multirow{4}{*}{$\begin{array}{c}\text { Paso } \\
1\end{array}$} & $\begin{array}{c}\text { Otra Facultad } \\
\text { Costo }<2\end{array}$ & 0,24 & 0,08 & $\begin{array}{l}8,857 \\
0,526\end{array}$ & $\begin{array}{l}1 \\
2\end{array}$ & $\begin{array}{c}0 \\
0,77\end{array}$ & 1,275 & 1,086 & 1,495 \\
\hline & Costo 2 - 3 & 0.11 & 0.15 & 0.48 & 1 & 0.49 & 1.11 & 0.826 & 1.494 \\
\hline & Costo > 3 & 0,09 & 0,17 & 0,258 & 1 & 0,61 & 1,088 & 0,785 & 1,509 \\
\hline & TIPO & 0,17 & 0,08 & 4,934 & 1 & 0,03 & 1,189 & 1,021 & 1,384 \\
\hline Paso & Fac salud & 0,25 & 0,07 & 13,54 & 1 & 0 & 1,277 & 1,121 & 1,455 \\
\hline 2 & Fumador & 0.18 & 0.08 & 5.226 & 1 & 0.02 & 1.194 & 1.026 & 1.39 \\
\hline
\end{tabular}

EE: Error estándar; EW: Estadístico de Wald; GL: Grados de libertad; S: significancia

\section{DISCUSIÓN}

Se realizó un estudio de seguimiento de los estudiantes matriculados en la Universidad Santiago de Cali en el segundo semestre de 2004, analizando su permanencia en los programas académicos según el hábito de fumar, programa académico y género, hasta el séptimo semestre, en 2007. Los datos de matrícula se obtuvieron de la Secretaría Académica de la Universidad y lo relacionado con el hábito de fumar, mediante la aplicación de una encuesta en los semestres 1, 3, 5 y 7. Según el análisis bivariado, las variables género y estado civil no presentaron asociaciones estadísticamente significativas con la supervivencia; el análisis multivariado excluyó la variable costos de matrícula. Los hallazgos de este estudio permiten concluir que tienen menor deserción de la universidad aquellos sin el hábito de fumar y que son estudiantes de un programa diferente a los de salud.

La relación del tabaquismo con el rendimiento escolar fue analizado por Anaya-Ocampo y colaboradores en un estudio realizado en Morelos (México) 
entre los años 1998 a 2001 quienes encontraron una vinculación del bajo desempeño escolar con la persistencia del tabaquismo (6). Una revisión del "Estado del Arte" sobre deserción estudiantil realizada en 2002 mediante convenio de la Universidad Nacional de Colombia y el Instituto Colombiano para el Fomento de la Educación Superior (ICFES) (11) analizó los diversos factores asociados a la deserción estudiantil de los programas académicos y llama la atención que no se contemplan los hábitos como el cigarrillo o el consumo de bebidas alcohólicas como factores a tener en cuenta en el análisis. Estos factores podrían estar incluidos en el factor denominado "adaptación social del estudiante con sus pares u homólogos".

En nuestro estudio, al séptimo semestre de los diferentes programas académicos permanecen en la universidad el $29.2 \%$ de los estudiantes matriculados el primer semestre. Rodríguez (12) en la Universidad Nacional de Colombia encontró que el $26 \%$ de los estudiantes terminan sus estudios al $10^{\circ}$ semestre. Al estudiar, mediante análisis de supervivencia la permanencia de los estudiantes en la universidad, comparando los no fumadores con fumadores habituales y con los esporádicos, se encontró que los fumadores habituales permanecen menos en la universidad que los no fumadores, con diferencias significativas. Este hallazgo confirma la influencia del cigarrillo sobre el rendimiento escolar, situación descrita por Hu y colaboradores (8) en un estudio en adolescentes y en el estudio de Anaya y colaboradores (6) en Morelos, México. Al considerar que el coeficiente intelectual de los fumadores es similar al de los no fumadores $(9,13)$, las explicaciones posibles de estos hallazgos se relacionan con el consumo simultáneo de otras sustancias psicoactivas por el fumador (alcohol, drogas) (14), mayor frecuencia de actividades extracurriculares sacrificando las académicas, entre otras. Sabia y colaboradores (15) encontraron que los fumadores de edad media, tienen mayor riesgo de pobre memoria que los no fumadores.

Al discriminar según la Facultad, se encontró que el hábito de fumar está asociado con la deserción en los "programas de no salud", no así en los programas de salud. Los estudiantes de la Facultad de salud fuman menos que los de "no salud" con diferencias significativas al ingreso a la universidad (19,8 salud vs 30,8 no salud) (2). El factor económico descrito por otros estudios (3) juega un papel importante en la diferencia existente entre los estudiantes de los programas de salud y los de otras áreas, así, el valor de la matrícula es aproximadamente tres veces superior en los programas de salud que en el resto. Esto puede explicar que en el análisis de las variables mediante la regresión de Cox, el costo de la matrícula no es una variable importante y se comporta igual que el 
tipo de programa académico. La exigencia académica es mayor y determina que los estudiantes de salud deban tener dedicación exclusiva a sus estudios no pudiendo realizar labores que les permitan obtener ingresos, lo cual no sucede con los otros programas académicos. Otro aspecto que puede estar relacionado con las diferencias en la prevalencia del hábito de fumar y en la supervivencia en la universidad es el conocimiento por los estudiantes de salud de los efectos nocivos del cigarrillo sobre la salud, los cuales no son tan evidentes en el resto de estudiantes.

Los hallazgos de este estudio podrían estar influenciados por sesgo de no respuesta por los estudiantes en los momentos de aplicación de la encuesta anualmente, especialmente en los alumnos recién ingresados a la universidad del área de la salud (1). No existen razones para pensar que los fumadores tendrían motivos para no responder, teniendo en cuenta que conocían de años anteriores el formulario y el responder la encuesta no tenía relación con la permanencia en la universidad.

Este estudio reafirma la necesidad de estudiar con detalle los efectos del cigarrillo sobre el rendimiento académico de los estudiantes de los programas de pregrado universitario. Es necesario que las autoridades universitarias actúen definiendo políticas dirigidas a disminuir el consumo de cigarrillo en los ambientes universitarios así como aplicando la Resolución 01956 de mayo de 2008 del Ministerio de la Protección Social la cual adopta medidas en relación con el consumo del tabaco en Colombia

\section{REFERENCIAS}

1. Tafur LA, Ordóñez G, Millán JC, Varela JM, Rebellón P. Tabaquismo en personal de la Universidad Santiago de Cali. Colombia Médica 2005;36: 194-198.

2. Tafur LA., Ordóñez G, Millán JC, Varela JM, Rebellón P. Prevalencia de tabaquismo en estudiantes recién ingresados a la Universidad Santiago de Cali. Colombia Médica 2006;37:126-132.

3. Sánchez F, Quirós M, Reverón C, Rodríguez A. Equidad Social en el Acceso y Permanencia en la Universidad Pública. Determinantes y Factores Asociados. Centro de Estudios para el Desarrollo Económico (CEDE), Universidad de los Andes, Bogotá; 2002.

4. Korhonen V, Laukkanen E, Peiponen S, Lehtonen J, Viinamäki H. Effect of major depression on the self-image of adolescent boys and girls. J Youth Adolesc. 2001; 30: 697-706.

5. Pérez N, Murillo R, Pinzón C, Hernández G. Costos de la atención médica del Cáncer de Pulmón, la EPOC y la IAM atribuibles al consumo de tabaco en Colombia. (proyecto multicéntrico de la OPS). Rev Colomb de cancerol 2007; 11(4):241-249.

6. Anaya R, Arillo E, Sánchez LM, Lazcano E. Bajo desempeño escolar relacionado con la persistencia del tabaquismo en una cohorte de estudiantes en México. Salud Pública Mex 2006;48 supl I:517-529. 
7. Caballero A, González B, Pinilla J, Barbet P. Factores predictores del inicio y consolidación del consumo de tabaco en adolescentes. Gac Sanit 2005; 19(6):440.7.

8. Hu T, Lin Z, Keeler TE. Teenage smoking, attempts to quit, and school perfomance. Am J Public Health. 1998;88:940-943.

9. WHO: building blocks for tobacco control - a handbook. World Health organization; 2004

10. Dietz K, Gail M, Krickeberg K, Singer B. Statistics in the Health Sciences. Editorial Springer. New York; 1996. p. 48-67.

11. Universidad Nacional de Colombia-ICFES. Estudio de la Deserción Estudiantil en la Educación Superior en Colombia. Convenio 107/2002. UN-ICFES. Bogotá; 2002.

12. Rodríguez J. Repitentes, Desertores y Universidad ¿Éxito Institucional y Fracaso Personal? Publicaciones Universidad Nacional de Colombia. Bogotá; 1987.

13. Matarazzo JD, Saslow G. Psycological and related characteristics of smokers and nonsmokers. Psychol Bull 1960:57:493-513.

14. Herrera M, Wagner FA, Velasco E, Borges G, Lazcano E. Inicio en el consumo de alcohol y tabaco y transición a otras drogas en estudiantes de Morelos, México. Salud Publica Mex 2004; 46:132-140.

15. Sabia S, Marmo M, Dufouil C, Singh A. Smoking History and Cognitive Function in Middle Age From the Whitehall II Study. Arch Intern Med. 2008;168(11):1165-1173. 